\title{
Self-Consistency and Calibration of Cluster Number Count Surveys for Dark Energy
}

\author{
Wayne $\mathrm{Hu}$ \\ Center for Cosmological Physics, Department of Astronomy and Astrophysics, \\ and Enrico Fermi Institute, University of Chicago, Chicago IL 60637
}

\begin{abstract}
Cluster number counts offer sensitive probes of the dark energy if and only if the evolution of the cluster mass versus observable relation(s) is well calibrated. We investigate the potential for internal calibration by demanding consistency in the counts as a function of the observable. In the context of a constant dark energy equation of state, known initial fluctuation amplitude expected from the $\mathrm{CMB}$, universal underlying mass function, and an idealized selection, we find that the ambiguity from the normalization of the mass-observable relationships, or an extrapolation of external massobservable determinations from higher masses, can be largely eliminated with a sufficiently deep survey, even allowing for an arbitrary evolution. More generally, number counts as a function of both the redshift and the observable enable strong consistency tests on assumptions made in modelling the mass-observable relations and cosmology.
\end{abstract}

\section{INTRODUCTION}

The number density of massive galaxy clusters is exponentially sensitive to the amplitude of initially Gaussian density fluctuations and has long been recognized as a sensitive cosmological probe 1]. In particular, the evolution of the number counts above a given mass threshold can determine the properties of the dark energy that accelerates the expansion [2, 3, 4].

This potential can only be realized if, in addition to the cluster redshifts, the cluster masses themselves are known, at least statistically. Unfortunately the total mass is not a direct observable and must be inferred through scaling relations with, e.g. the SunyaevZel'dovich (SZ) flux decrement, the $X$-ray flux or temperature, weak lensing shear, or optical velocity dispersion. Indeed the normalization of the mass-temperature relation is currently the leading source of ambiguity in interpreting the local cluster abundance (e.g. [5, 6]). It compromises dark energy constraints if the cluster mass selection cannot be defined to a few percent in mass [3].

The mass-observable relation can potentially be calibrated within a survey itself if its effect on the number counts is not degenerate with the cosmology. For example, with a single cut on the temperature, the survey itself can calibrate the mass-temperature normalization to better than the tens of percent that span the determinations in the current literature, if it does not evolve [7]. However this method fails if the relationship has uncertain evolution that mimics the cosmology [8].

Because the number density of clusters as a function of mass has a fixed functional form given by cosmological simulations (e.g. 9, 10]), cluster number counts as a function of the observable in principle have the ability to self-calibrate even an evolving mass-observable relation. Here we study the potential for internal calibration and consistency checks in idealized future cluster number count surveys.

\section{STATISTICAL FORECASTS}

The cosmological utility of cluster counts stems from the simulation-based prediction of their comoving differential number density as a function of mass [9],

$$
\frac{d \bar{n}}{d \ln M}=0.3 \frac{\rho_{m}}{M} \frac{d \ln \sigma^{-1}}{d \ln M} \exp \left[-\left|\ln \sigma^{-1}+0.64\right|^{3.82}\right]
$$

Here $\sigma^{2}(M, z)$ is the variance in the linear density field smoothed with a top hat that encloses $M$ at the mean matter density today $\rho_{m}$. The cosmological sensitivity comes from these quantities and the comoving volume element in a redshift slice and solid angle.

To assess the sensitivity of counts to various cosmological and mass-observable scaling parameters $p_{\alpha}$, we employ the Fisher matrix technique 11, 12.

$$
F_{\alpha \beta}=\sum_{i j} \frac{\partial \bar{n}_{i}}{\partial p_{\alpha}}\left(\mathbf{C}^{-1}\right)_{i j} \frac{\partial \bar{n}_{j}}{\partial p_{\beta}}
$$

where the covariance matrix is given by

$$
C_{i j}=\left(\left\langle n_{i} n_{j}\right\rangle-\bar{n}_{i} \bar{n}_{j}\right)+\delta_{i j} \bar{n}_{i} / V_{i}
$$

The first term represents sample covariance in the volume $V_{i}$ from large-scale structure, calculated as described in [1] , and the second term shot variance. Here the number density in a bin $n_{i}$ is defined by the redshift interval $\Delta z$ around $z_{i}$ and a selection based on some observable quantity $f$, such as the SZ flux decrement. Note that sampling errors for the selections in the same redshift bin completely covary.

The Fisher matrix is a local approximation to the covariance matrix of the parameters

$$
\mathbf{C}_{\text {tot }}=\left(\mathbf{F}+\mathbf{C}_{\text {prior }}^{-1}\right)^{-1},
$$

where $\mathbf{C}_{\text {prior }}$ is the covariance matrix from prior information. 
The Fisher matrix is evaluated around a fiducial choice of parameters. For the cosmology, we take: the dark energy density $\Omega_{\mathrm{DE}}=0.65$, equation of state $w=-1$, physical matter density $\Omega_{m} h^{2}=0.148$, physical baryon density $\Omega_{b} h^{2}=0.02$, tilt $n_{s}=1$, and the initial normalization of the curvature fluctuations $\delta_{\zeta}=4.79 \times 10^{-5}$ at $k=0.01 \mathrm{Mpc}^{-1}[13]$ (more conventionally: $\delta_{H}=$ $4.42 \times 10^{-5}, \sigma_{8}=0.92$, or $\left.M_{*}=1.2 \times 10^{13} h^{-1} M_{\odot}\right)$. We will however work in a future context where the cosmic microwave background (CMB) has constrained the high redshift universe parameters with $1 \sigma$ errors of $\sigma\left(\ln \Omega_{m} h^{2}\right)=\sigma\left(\ln \Omega_{b} h^{2}\right)=\sigma\left(n_{s}\right)=\sigma\left(\ln \delta_{\zeta}\right)=0.01$ (e.g. [13] ) in a flat universe, leaving the dark energy parameters to be determined by the survey.

For the mass-observable relation, we take the form

$$
\frac{M_{f}}{M_{0}}=e^{A(z)}\left(\frac{f}{f_{0}}\right)^{p(z)}
$$

where $M_{0}$ and $f_{0}$ are dimensional constants and the normalization parameter $A(z)$ is dimensionless. We take the scaling parameters as the amplitudes of a piecewise constant form $A\left(z_{i}\right)$ and $p\left(z_{i}\right)$. Specifically, given a variation $\delta A\left(z_{i}\right)$ and $\delta \ln p\left(z_{i}\right)$ from the fiducial model, the true mass is related to the apparent mass as

$$
\ln M=\ln M_{f}+\delta A\left(z_{i}\right)+\delta \ln p\left(z_{i}\right) \ln \left(\frac{M_{f}}{M_{p}}\right),
$$

where $M_{p}=M_{0} e^{A}$ is the pivot mass scale that defines a normalization point for possible external information.

This generalizes previous treatments which have assumed constant normalization [7] and power law evolution [8]. In the limit of small redshift bins, these simpler cases can be recovered from our more general treatment by noting that under a re-parameterization of the space to the set $\pi_{\mu}\left(p_{\alpha}\right)$, possibly of lower dimension, the covariance matrix transforms as

$$
C_{\mu \nu}=\sum_{\alpha \beta} \frac{\partial \pi_{\mu}}{\partial p_{\alpha}} C_{\alpha \beta} \frac{\partial \pi_{\nu}}{\partial p_{\beta}}
$$

The Fisher matrix definition itself can be so viewed.

In the usual approach (e.g. 3] ), all of the clusters in a given redshift bin above a given observable threshold are simply binned together. Clearly, allowing for arbitrary variations $\delta A\left(z_{i}\right)$ no cosmological information can be extracted. However the data at a given redshift may be binned into several apparent mass bins based on the observable $f$. The additional information supplied by multiple bins allows for a breaking of the degeneracy.

Likewise, even if the normalization is fixed by detailed followup by $X$-ray or weak lensing measurements at some mass $M_{p}$ (e.g. [8, 14]), an uncertain scaling index $\delta p\left(z_{i}\right)$ would again destroy the cosmological information with a single bin if $M_{p} \neq M_{f, \min }$. Multiple bins again in principle allow the cosmology and the mass-observable relations to be jointly determined.

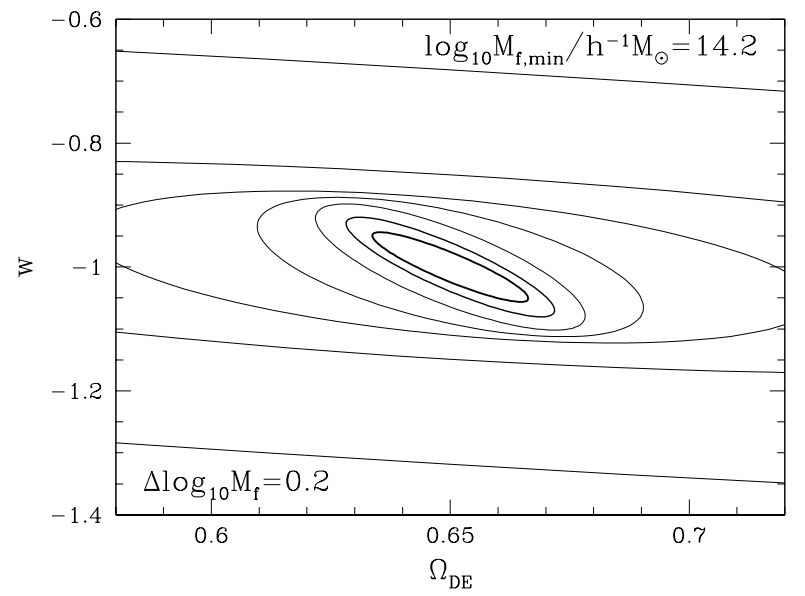

FIG. 1: Consistency test with differential counts. Division of the number counts into apparent mass bins from a minimum of $\log _{10} M_{f, \min } / h^{-1} \mathrm{M}_{\odot}=14.2$ upwards in steps of 0.2 (outwards in ellipses) allows several nearly independent constraints on the dark energy that test consistency with the assumed mass-observable relation. Strong priors are assumed on the high redshift cosmology (see text).

This treatment ignores intrinsic scatter in the massobservable relation as well as measurement error in the observable. More realistically the selection function is not sharp in mass but assuming its functional form is well characterized this does not substantially compromise the cosmological information [7, 15].

For definiteness, let us take a fiducial cluster survey with specifications similar to the planned South Pole Telescope (SPT) Survey for clusters with the SZ effect: an area of $4000 \mathrm{deg}^{2}$ survey and a sensitivity corresponding to a constant $\log _{10} M_{f, \min } / h^{-1} M_{\odot}=14.2$ (e.g. [8]). We divide the number counts into bins of redshift $\Delta z=$ 0.1 out to $z_{\max }=3$ and $\Delta \log _{10} M_{f}=0.43 \Delta \ln M_{f}=0.2$. This crude binning is sufficient to retain the cosmological information. For illustration purposes, we take a pivot mass scale of $\log _{10} M_{p} / h^{-1} M_{\odot}=14.7$ to reflect potential mass measurement followup on the high mass end.

\section{CONSISTENCY AND CALIBRATION}

Let us first consider that simulations or mass followup with different observables have placed strong priors on the mass-observable normalization and scaling in$\operatorname{dex} \sigma\left(\delta A\left(z_{i}\right)\right)=\sigma\left(\delta \ln p\left(z_{i}\right)\right)=0$. In this case, the division into mass bins yields nearly independent measurements of the dark energy for a consistency check.

In Fig. 1] we illustrate the consistency check in the $\left(\Omega_{\mathrm{DE}}, w\right)$ plane for individual mass bins of $\Delta \log _{10} M_{f}=$ 0.2 from $\log \left(M_{f, \min } / h^{-1} M_{\odot}\right)=14.2$. Although the strongest constraints do come from the lowest mass bin, as expected from the increasing rarity of massive clusters at high redshift, the first 6 bins show significant constraints on the dark energy. This consistency test 


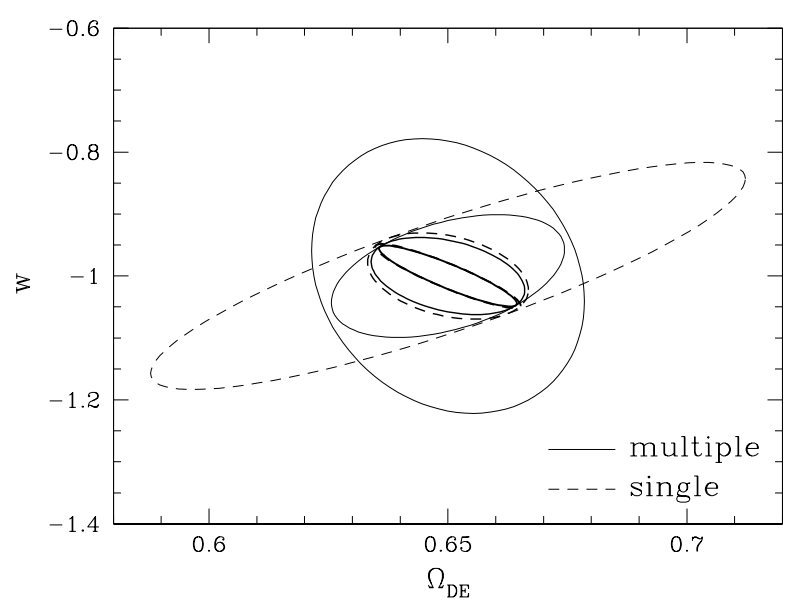

FIG. 2: Normalization self-calibration and the $68 \%$ CL dark energy constraints. Solid curves represent binning as in Fig. 1 d dashed curves represent a single bin with threshold $M_{f \text {,min }}$. Inner to outer ellipses: fixed, constant, power law evolution, arbitrary amplitude $\delta A\left(z_{i}\right)$ (infinite for single mass bin). Strong priors on the scaling index $p$ are assumed.

demonstrates that there is sufficient information in the mass binning to attempt some degree of self-calibration of the mass-observable relations.

Let us next keep the scaling index fixed $p\left(z_{i}\right)$ but allow the normalization $A\left(z_{i}\right)$ to vary (see Fig. [2). Solid lines represent constraints with the 6 mass bins and dashed lines those with a single mass bin or mass threshold. We first allow only a redshift-independent normalization factor $\delta A\left(z_{i}\right)=\delta A_{0}$. As shown in [7], even a single mass bin is sufficient to calibrate the relation to good enough accuracy for dark energy constraints (here $\sigma(\delta A)=0.05$ ). We next employ a power law evolution in the normalization $\delta A\left(z_{i}\right)=\delta A_{0}+n_{a} \ln \left(1+z_{i}\right)$. Here errors on the dark energy parameters degrade substantially with a single mass bin [8], but much of the loss is recovered from the multiple binning. Finally, we take a fully arbitrary evolution in the redshift bins, i.e. no constraint on the form of $\delta A\left(z_{i}\right)$. As expected, there is no constraint on the dark energy with a single mass bin, but with multiple bins interesting constraints on the dark energy can still be extracted. The key here is that the dark energy evolution is assumed to be smooth and parameterized by two numbers and so cannot compensate an arbitrary variation in the normalization evolution.

Figure 3 (top) shows the errors on $\delta A\left(z_{i}\right)$ with roughly $10 \%$ constraints on the normalization in each redshift band out to $z \sim 2$. Note that the covariance between the bands is nearly complete so that the overall normalization is still only known to $\sim 5 \%$ but that the evolution is highly constrained. Conversely, external information on the mass-observable normalization would have to be substantially better than $10 \%$ to improve on the internal results. In the context of our fiducial cosmology, the critical prior assumption is that the initial amplitude of the fluctuations is fixed by the CMB. Otherwise what is con- strained by the multiple mass bins is a degenerate combination of the initial amplitude and the mass-observable normalization.

We have so far assumed that the scaling index $p(z)$ is known a priori but of course that too is part of the uncertain mass-observable relation. Allowing for arbitrary variations $\delta A\left(z_{i}\right)$ and $\delta \ln p\left(z_{i}\right)$ destroys most of the information on the dark energy. However in the less drastic cases of adding a constant $\delta \ln p$ to a constant $\delta A$ for a two parameter model, the additional degradation $\sigma(w)$ is a negligible $3 \%$ and of the addition of power law variation in $p(z), \delta \ln p=\delta \ln p_{0}+n_{p} \ln \left(1+z_{i}\right)$ to power law variation in $\delta A$ for a 4 parameter model, a factor of 1.76 for a total of $\sigma(w)=0.12$.

Another interesting case to consider is if some independent mass calibration, say from weak lensing or $X$-ray temperature followup, normalizes the mass-observable relation on the high mass end. Extrapolation down to the survey mass limit can be dangerous due to the uncertain physics of low mass clusters. For illustrative purposes, let us take this constraint as $\sigma\left(A\left(z_{i}\right)\right)=0.1$ at the pivot mass of $\log _{10} M_{p} / h^{-1} M_{\odot}=14.7$. Despite the constraint on the normalization, if we allow for arbitrary variations in the scaling index and retain only a threshold at $>14.2$, no constraint on the dark energy can be extracted. With multiple bins, the errors on $w$ are only degraded by a factor of 1.16 to $\sigma(w)=0.09$ for a completely arbitrary $\delta \ln p\left(z_{i}\right)$. Furthermore the errors on $\delta \ln p$ shown in Fig. 3 (bottom) are at the several percent level and would be of interest in studying cluster physics.

\section{DISCUSSION}

As in the case of classical cosmological tests for the dark energy involving standardized candles and rulers, e.g. supernovae and the peaks in the CMB power spectrum, cluster number count tests require a standardized mass based on observable quantities. We have demonstrated that consistency with the well-determined shape of the mass function from cosmological simulations can in principle be used to calibrate the survey internally. Two examples are a normalization that has an arbitrary evolution in redshift and a scaling from the more easily calibrated high mass end that has an arbitrary evolution in the index.

Our study involves a number of idealizations that merit future study and so internal calibration is best viewed as a useful check on cross-calibration studies with detailed multi-wavelength followup (e.g. [8, 16]) and cosmological simulations. The crucial assumptions are that the survey be sufficiently deep to explore a substantial dynamic range in the mass function, the underlying mass function is known given a cosmology, the mass-observable selection function is sharp compared with the binning, the mean mass-observable relation is power law in form, the high redshift normalization of the fluctuations is fixed by future CMB data, and the dark energy equation of state 


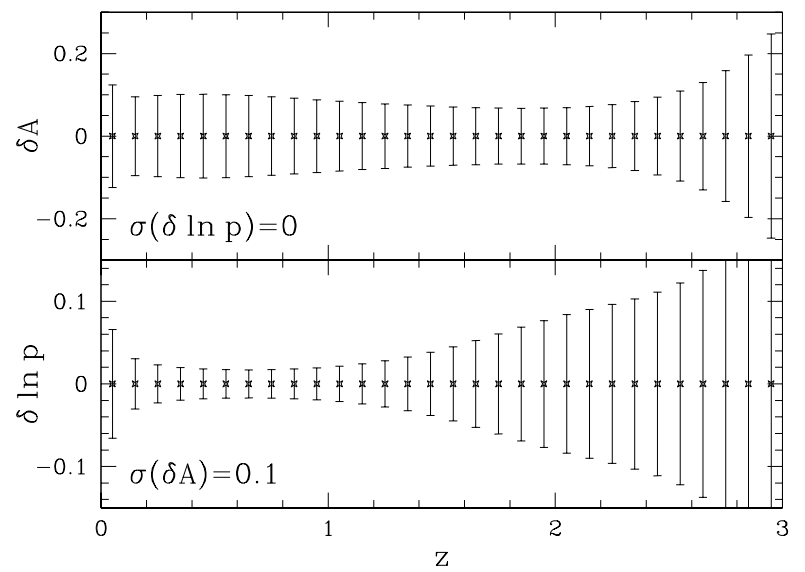

FIG. 3: Errors on scaling parameters. Top: errors on $\delta A\left(z_{i}\right)$ assuming fixed scaling index $p$ and the apparent mass divisions of Fig. 2 Bottom: errors on $\delta \ln p\left(z_{i}\right)$ assuming a prior on the mass-observable relation $\sigma\left(\delta A\left(z_{i}\right)\right)=0.1$ from mass followup at $\log _{10} M_{p} / h^{-1} \mathrm{M}_{\odot}=14.7$

is constant.

In the fiducial model, a depth comparable to that planned for the SPT SZ survey is sufficient to make selfcalibration useful but note that the necessary mass limit scales roughly with the non-linear mass scale $M_{*}$. That depends on the currently uncertain normalization and so if the normalization is lower than our fiducial choice it may be beneficial to sacrifice survey width for depth.

While the mass function form is based on simulations and currently its scaling with cosmology is only known to the $\sim 10 \%$ level $[9]$, determination requires only the wellunderstood gravitational physics of the dark matter and is far more secure than simulation-based mass-observable relations. In any case, this knowledge is a prerequisite for any cluster number count study of cosmology.
We have assumed a deterministic mass-observable relation that makes the selection function sharp in apparent mass. Gaussian scatter in this relation does not seriously compromise the cosmological information 7, 15] but long uncharacterized tails to low mass would, given the steep mass function. Likewise the cluster physics of preheating and cooling can enter into the low mass end to make the mass-observable relations deviate from a power law. Here the binning serves as a useful consistency test for the implicitly assumed cluster physics.

The required prior information on the initial normalization is within reach of the upcoming CMB satellite missions if the extent of reionization can be determined (e.g. 13, 17]). If not, binning still serves to constrain the evolution of the mass-observable relation but leaves a degeneracy between the overall normalization of the relations and the fluctuations which can be fixed, e.g. by CMB lensing, Ly $\alpha$ forest clustering or even local cluster abundance studies. Conversely, external calibration of the mass-observable relation at the percent level can determine the fluctuation normalization at a comparable level. Binning can then provide tests of the dark energy model assumptions [18] or other cosmological priors. It is especially valuable if redshifts are available only locally (e.g. $z_{\max } \lesssim 0.6$ from current optical surveys).

In summary, utilizing the extra information in counting clusters as a function of both the redshift and their observable properties allows for joint solutions to the massobservable relations and cosmology, if they are both simple. If not, it offers valuable internal consistency checks against overly simplistic assumptions.

Acknowledgments: I thank Z. Haiman, A.V. Kravtsov, J.J. Mohr, C. Pryke and the Chicago Thunch group for useful conversations and J. Valdes for *nix expertise in file recovery. WH is supported by NASA NAG5-10840 and the DOE OJI program.
[1] A.E. Evrard, Astrophys. J, 341, L71 (1989); C.S. Frenk, S.D.M. White, G. Efstathiou, M. Davis, ibid, 351, 10 (1990); J.P. Henry, M. Arnaud, ibid, 372, 410 (1991); P.B. Lilje, ibid, 386, L33 (1992); N.A. Bahcall, R.Y. Cen, ibid, 565, L5 (1992).

[2] L. Wang, P.J. Steinhardt, Astrophys. J, 508, 483 (1998).

[3] Z. Haiman, J.J. Mohr, G.P. Holder, Astrophys. J, 553, 545 (2001).

[4] J.M. Diego, E. Martinez-Gonzalez, J.L. Sanz, N. Benitez, J. Silk, Mon. Not. R. Astron. Soc., 331, 556 (2001).

[5] E. Pierpaoli, D. Scott, M. White, Mon. Not. R. Astron. Soc., 325, 77 (2001).

[6] U. Seljak, Mon. Not. R. Astron. Soc., 337, 769 (2002).

[7] E.S. Levine, A.E. Schulz, M. White, Astrophys. J, submitted, astro-ph/0204273 (2002).

[8] S. Majumdar, J.J. Mohr, Astrophys. J, submitted, astroph/0208002 (2002).

[9] A. Jenkins, et al. MNRAS, 321, 372 (2001).
[10] D. Reed, et al. Mon. Not. R. Astron. Soc., submitted, astro-ph/0301270 (2003).

[11] W. Hu, A.V. Kravtsov, Astrophys. J, in press, astroph/0203169 (2003).

[12] G. Holder, Z. Haiman, J.J. Mohr, Astrophys. J Lett., 560, 111 (2001).

[13] W. Hu, Phys. Rev. D, 65, 023003 (2002).

[14] D. Huterer, M. White, Astrophys. J Lett., 578, L95 (2002).

[15] G.P. Holder, J.J. Mohr, J.E. Carlstrom, A.E. Evrard, E.M. Leitch, Astrophys. J, 544, 629 (2000).

[16] L. Verde, Z. Haiman, D.N. Spergel, Astrophys. J, 581, 5 (2002).

[17] M. Kaplinghat, et al. Astrophys. J, in press, astro$\mathrm{ph} / 0207591$ (2002).

[18] J. Weller, R. Battye, R. Kneissl, Phys. Rev. Lett., 88, 231301 (2002). 